\title{
Association and Principal Component Analyses of Eating Quality Traits of 141 Japonica Rice Cultivars in China
}

\author{
Lixia Zhang, Ying Yan, Zejun Hu, Kai Wang, Liming Cao, Shujun Wu* \\ Crop Breeding and Cultivation Research Institute, Shanghai Academy of Agricultural Sciences, Shanghai, China \\ Email address: \\ zhanglixia1@saas.sh.cn (Lixia Zhang), yanying@saas.sh.cn (Ying Yan), huzejun@saas.sh.cn (Zejun Hu), wangkai@saas.sh.cn (Kai Wang), \\ caoliming@saas.sh.cn (Liming Cao),136601095@qq.com (Shujun Wu),wushujun@saas.sh.cn (Shujun Wu) \\ ${ }^{*}$ Corresponding author
}

\section{To cite this article:}

Lixia Zhang, Ying Yan, Zejun Hu, Kai Wang, Liming Cao, Shujun Wu. Association and Principal Component Analyses of Eating Quality Traits of 141 Japonica Rice Cultivars in China. American Journal of Agriculture and Forestry. Vol. 9, No. 1, 2021, pp. 37-41.

doi: 10.11648/j.ajaf.20210901.16

Received: January 5, 2021; Accepted: January 19, 2021; Published: January 30, 2021

\begin{abstract}
Eating quality is the important trait of rice, and rice breeders also employ eating quality in breeding selection for advanced generations. Therefore, to better understand the factors affecting the eating quality of japonica rice in China, the physicochemical indices [apparent amylose content (AAC), gel consistency (GC), protein content (PC), and gelatinization temperature (GT)], rapid visco analyzer parameters, and cooked rice taste values of 141 rice cultivars were analyzed. The rice cultivars were divided into low, medium, and total AAC groups. Association and principal component analyses of these indices were performed. In total AAC rice, cooked rice taste value showed a significant negative correlation with AAC, final viscosity (CPV), setback viscosity (SBV), consistence viscosity (CSV), and a positive correlation with GC, peak viscosity (PKV), holding strength (HPV), and breakdown viscosity (BDV). PC showed a significant negative correlation with cooked rice taste value in low, medium, and total AAC rice. For both low and medium $\mathrm{AAC}$ rice, the factor load matrix of $\mathrm{PC}$ and cooked rice taste value in factor 1 was remarkably higher than that of the other indices, indicating that $\mathrm{PC}$ was the most important factor for the eating quality of low and medium AAC rice. For total AAC rice, the factor load matrix of AAC, GC, and cooked rice taste value in factor 1 was remarkably higher than that of the other indices, indicating that $\mathrm{AAC}$ and $\mathrm{GC}$ were important factors for the eating quality of different type rice cultivars. The results showed that AAC and GC were more important for the eating quality of different type rice cultivars, while PC was more important for the eating quality of similar type rice cultivars. Our findings offer new insight into target traits in breeding rice with high eating quality.
\end{abstract}

Keywords: Japonica Rice, Eating Quality, Apparent Amylose Content, Protein Content

\section{Introduction}

Rice (Oryza sativa L.) is one of the most widely consumed foods in the world, and the subspecies japonica is commonly consumed in the Yangtze River Delta Region and North China. Eating quality is the most important rice quality for consumers and primarily affected by hardness, stickiness, appearance and taste [1].

Physicochemical indices, such as apparent amylose content (AAC), gel consistency (GC), protein content (PC), and gelatinization temperature (GT) have been widely studied to evaluate rice eating quality $[2,3]$. Rice with low AAC and PC becomes soft and sticky after cooking $[4,5]$. Recently, cooked rice taste analyzers and Rapid Visco Analyzers (RVAs) have been widely used by breeders to evaluate rice eating quality [6, 7].

The eating quality of rice is highly complex [8]. Although the relationships among the physicochemical indices, pasting properties, and eating quality have been widely studied $[9,10]$, their correlations have not been studied in detail as different groups by multivariate analysis. Recently, low AAC rice has been developed and is preferred in the Yangtze River Delta Region because of its high eating quality. To better understand the factors affecting the eating quality of Japonica rice in China, the physicochemical indices, RVA parameters, and cooked taste values of 141 japonica rice cultivars were 
measured. The cultivars were divided into low, medium, and total AAC rice groups. Association and principal component analyses of the eating quality indices were performed in all three groups. The results will be useful for breeders to select high eating quality rice.

\section{Materials and Methods}

\subsection{Plant Materials and Sample Preparation}

Rice plants were grown in the Zhuanghang experiment field (Shanghai, China) in May 2018 and were harvested in November 2018. The rice seeds were air-dried to $\sim 14 \%$ moisture content. Brown rice grains were husked, polished, milled, and then the flour was passed through 100-mesh sieves.

\subsection{Measurement of Physicochemical Properties}

$\mathrm{AC}$ and GC was determined as previously described [11]. Nitrogen was determined using an Automatic Kjeldahl Nitrogen Determination Apparatus (8400, Foss, Hillerød, Denmark). The total PC was obtained based on the nitrogen by multiplying a nitrogen protein conversion factor of 5.95. The alkali spreading value (ASV), an indicator of GT, was measured as previously described [12].

\subsection{Rice Eating Quality Analysis}

The taste value of cooked rice was analyzed with a rice taste meter (STA1B, SATAKE Co. Ltd., Japan).

\subsection{Rice Pasting Property Analysis of Starch}

Rice pasting properties were determined using a RVA (Newport Science, Warriewood, Australia) and the values were analyzed by Thermocline for Windows v3 [13]. RVA profile was expressed by pasting temperature (Pat), peak viscosity (PKV); final viscosity (CPV), holding strength (HPV), breakdown viscosity (BDV), setback viscosity (SBV), consistence viscosity (CSV). The unit used was "Rapid Visco Units" (RVU).

\subsection{Statistical Analysis}

All samples were analyzed in three technical replicates. Association and principal component analysis were performed using SPSS 18. Pearson's correlation coefficient was calculated to analyze the interrelationship among the parameters of rice eating quality. The results were analyzed using Tukey's tests at $p<0.05$ significance level.

\section{Results and Discussion}

\subsection{Distribution of Physicochemical Indices, $R V A$ Parameters, and Taste Values}

The physicochemical indices, rapid visco analyzer parameters, and cooked taste values of 141 rice cultivars were analyzed and the rice cultivars were divided into low, medium, and total (low and medium) AAC groups to compare the factors affecting the eating quality within similar and different type rice cultivars. The range distribution of the physicochemical indices, RVA parameters, and cooked rice taste values of low and medium AAC rice is listed in Tables $1-2$. The AAC range of low AAC rice was $4.7 \%-10.7 \%$, and that of medium AAC rice was $13.1 \%-18.5 \%$. The average GC of low and medium $\mathrm{AAC}$ rice was 82.7 and $70.6 \mathrm{~mm}$, respectively. The average $\mathrm{PC}$ of both low and medium AAC rice was $\sim 8.0 \%$. The average ASV of low and medium AAC rice was 5.1 and 5.5 grade. For the RVA parameters, the HPV, $\mathrm{CPV}, \mathrm{SBV}$, and CSV of low AAC rice was considerably lower than those of medium $\mathrm{AAC}$ rice, and the $\mathrm{BDV}$ of low $\mathrm{AAC}$ rice was remarkably higher than that of medium $\mathrm{AAC}$ rice. The average Pat of both low and medium AAC rice was $\sim 71{ }^{\circ} \mathrm{C}$. The average cooked rice taste values of low and medium AAC rice were 77.5 and 68.3 points, respectively. The kurtosis of Pat, AAC, and GC was high in low AAC rice, and that of SBV, CSV, and Pat was high in medium AAC rice. The skewness of Pat and ASV was high in low AAC rice, and that of SBV, Pat, and ASV was high in medium AAC rice. The coefficient of variation for SBV in the groups was high, indicating that variation was considerable between different cultivars within each group, especially in medium AAC rice.

Table 1. Distribution of physicochemical indices, RVA parameters, and cooked taste values of low AAC rice cultivars.

\begin{tabular}{|c|c|c|c|c|c|c|c|}
\hline & $\begin{array}{l}\text { Average } \\
\text { value }\end{array}$ & $\begin{array}{l}\text { Standard } \\
\text { deviation }\end{array}$ & Kurtosis & Skewness & $\begin{array}{l}\text { Minimal } \\
\text { value }\end{array}$ & $\begin{array}{l}\text { Maximum } \\
\text { value }\end{array}$ & $\begin{array}{l}\text { Coefficient of } \\
\text { variation }\end{array}$ \\
\hline AAC (\%) & 8.48 & 1.03 & 2.74 & -0.91 & 4.70 & 10.70 & 0.12 \\
\hline $\mathrm{GC}(\mathrm{mm})$ & 82.67 & 8.23 & 1.71 & 0.58 & 63.50 & 109.00 & 0.10 \\
\hline PC (\%) & 8.00 & 0.50 & -0.33 & -0.32 & 6.68 & 8.95 & 0.06 \\
\hline ASV (grade) & 5.08 & 1.04 & 0.68 & -1.15 & 2.00 & 6.92 & 0.20 \\
\hline Pat $\left({ }^{\circ} \mathrm{C}\right)$ & 70.51 & 2.31 & 4.00 & 2.00 & 67.45 & 78.65 & 0.03 \\
\hline PKV (RVU) & 261.87 & 36.47 & 0.17 & 0.72 & 194.08 & 349.83 & 0.14 \\
\hline HPV (RVU) & 126.25 & 24.12 & 0.21 & 0.05 & 59.25 & 186.17 & 0.19 \\
\hline CPV (RVU) & 177.88 & 26.63 & 1.07 & -0.30 & 90.67 & 240.67 & 0.15 \\
\hline BDV (RVU) & 135.62 & 25.29 & 0.26 & 0.76 & 94.08 & 201.67 & 0.19 \\
\hline SBV (RVU) & -83.99 & 27.75 & -0.26 & -0.54 & -149.08 & -37.50 & -0.33 \\
\hline CSV (RVU) & 51.63 & 5.84 & 1.13 & -0.87 & 31.42 & 60.08 & 0.11 \\
\hline Taste value (points) & 77.46 & 4.43 & -0.57 & 0.04 & 68.67 & 87.67 & 0.06 \\
\hline
\end{tabular}

Note: $\mathrm{n}=70$. 


\subsection{Correlation Coefficient of Physicochemical Indices}

AAC, GC, PC, and ASV were the important physicochemical indices of the rice eating quality, and their correlation coefficient is analyzed (Tables 3-5). For both low and medium AAC rice, the correlation between all indices was not significant. For the total AAC rice, AAC was significantly negatively correlated with GC.
AAC is considered to be the most crucial factor affecting eating quality $[14,15]$, but rice varieties with similar AAC often have different eating qualities [16, 17]. Herein, AAC showed a significant negative correlation with GC in total $\mathrm{AAC}$ rice but not in low or medium AAC rice, indicating that GC may evidently vary between different rice types.

Table 2. Distribution of physicochemical indices, RVA parameters, and taste values of medium AAC rice cultivars.

\begin{tabular}{|c|c|c|c|c|c|c|c|}
\hline & $\begin{array}{l}\text { Average } \\
\text { value }\end{array}$ & $\begin{array}{l}\text { Standard } \\
\text { deviation }\end{array}$ & Kurtosis & Skewness & $\begin{array}{l}\text { Minimal } \\
\text { value }\end{array}$ & $\begin{array}{l}\text { Maximum } \\
\text { value }\end{array}$ & $\begin{array}{l}\text { Coefficient of } \\
\text { variation }\end{array}$ \\
\hline $\mathrm{AAC}(\%)$ & 16.02 & 1.05 & 1.22 & -0.64 & 13.05 & 18.50 & 0.07 \\
\hline $\mathrm{GC}(\mathrm{mm})$ & 70.59 & 7.87 & 0.56 & -0.67 & 49.50 & 87.50 & 0.11 \\
\hline PC (\%) & 8.02 & 0.63 & 0.38 & -0.10 & 6.62 & 9.65 & 0.08 \\
\hline ASV (grade) & 5.52 & 0.99 & 1.19 & -1.13 & 2.33 & 6.92 & 0.18 \\
\hline Pat $\left({ }^{\circ} \mathrm{C}\right)$ & 71.12 & 1.56 & 1.71 & 1.14 & 68.75 & 76.55 & 0.02 \\
\hline PKV (RVU) & 235.73 & 21.78 & 0.70 & 0.13 & 173.58 & 297.83 & 0.09 \\
\hline CPV (RVU) & 247.11 & 20.12 & 0.29 & -0.14 & 190.33 & 293.92 & 0.08 \\
\hline BDV (RVU) & 78.63 & 18.76 & 1.09 & 0.49 & 41.42 & 145.50 & 0.24 \\
\hline SBV (RVU) & 11.38 & 20.56 & 6.71 & -1.86 & -87.17 & 44.50 & 1.81 \\
\hline CSV (RVU) & 90.01 & 8.23 & 3.15 & -1.00 & 58.33 & 109.92 & 0.09 \\
\hline Taste value (points) & 68.25 & 6.05 & 1.52 & 0.61 & 56.33 & 90.00 & 0.09 \\
\hline
\end{tabular}

Note: $\mathrm{n}=71$.

Table 3. Correlation coefficient of physicochemical indices of low AAC rice.

\begin{tabular}{llll}
\hline & AAC & GC & PC \\
\hline GC & -0.213 & & \\
PC & -0.118 & -0.077 & \\
ASV & $-0.283^{*}$ & 0.156 & -0.202 \\
\hline
\end{tabular}

Note: $\mathrm{n}=70 .{ }^{* *}$, significantly correlated at $0.01 \mathrm{level}$; *, significantly correlated at 0.05 level.

Table 4. Correlation coefficient of physicochemical indices of medium AAC rice.

\begin{tabular}{llll}
\hline & AAC & GC & PC \\
\hline GC & 0.029 & & \\
PC & -0.199 & 0.113 & \\
ASV & -0.033 & $-0.245^{*}$ & 0.076 \\
\hline
\end{tabular}

Note: $\mathrm{n}=71 . * *$, significantly correlated at 0.01 level; $*$, significantly correlated at 0.05 level.

Table 5. Correlation coefficient of physicochemical indices of total AAC rice.

\begin{tabular}{llll}
\hline & AAC & GC & PC \\
\hline GC & $-0.604^{* *}$ & & \\
PC & -0.053 & 0.032 & \\
ASV & 0.165 & -0.159 & -0.042 \\
\hline
\end{tabular}

Note: $\mathrm{n}=141 . * *$, significantly correlated at 0.01 level; $*$, significantly correlated at 0.05 level.

\subsection{Correlation Among Physicochemical Indices, RVA Parameters, and Cooked Rice Taste Values}

The correlations among cooked rice taste values, physicochemical indices, and RVA parameters were analyzed (Table 6). RVA characteristics are controlled mostly by $W x$ gene [18], which determines the eating quality by affecting $\mathrm{AAC}$ and $\mathrm{GC}$ [15]. In total AAC rice, cooked rice taste value showed a significant negative correlation with AAC, CPV, $\mathrm{SBV}$, and CSV, and a positive correlation with GC, PKV, HPV, and BDV; no such correlation was observed in low or medium $\mathrm{AAC}$ rice groups, indicating that these parameters may be important reference indices for different type rice cultivars. ASV and Pat are indicators of GT. The two indices were not correlated with cooked rice taste value in low, medium, and total AAC rice.

PC also affects eating quality [19]. PC showed a significant negative correlation with cooked rice taste value in low, medium, and total $\mathrm{AAC}$ rice, indicating that $\mathrm{PC}$ can be an essential index for evaluating eating quality in similar or different type rice cultivars. For different type rice cultivars, AAC, GC, and RVA parameters can be important indices for evaluating eating quality.

Table 6. Correlation coefficient between physicochemical indices and cooked rice taste value.

\begin{tabular}{|c|c|c|c|c|c|c|c|c|c|c|}
\hline AAC & GC & PC & ASV & Pat & PKV & HPV & CPV & BDV & SBV & CSV \\
\hline \multicolumn{11}{|c|}{ Low AAC rice } \\
\hline $\begin{array}{l}0.107 \\
\text { Medium }\end{array}$ & $\begin{array}{l}0.203 \\
\text { rice }\end{array}$ & $-0.373 * *$ & 0.022 & -0.071 & -0.028 & -0.221 & $-0.248^{*}$ & 0.170 & -0.200 & -0.215 \\
\hline 0.086 & -0.128 & $-0.395 * *$ & -0.070 & -0.178 & 0.096 & $0.278^{*}$ & 0.060 & 0.196 & 0.086 & -0.128 \\
\hline $\begin{array}{l}\text { Total AA } \\
-0.614^{* *}\end{array}$ & $0.402 * *$ & $-0.297 * *$ & -0.158 & -0.126 & $0.346^{* *}$ & $0.367 * *$ & $-0.563 * *$ & $0.584 * *$ & $-0.649 * *$ & $-0.669 * *$ \\
\hline
\end{tabular}

**, significantly correlated at 0.01 level; $*$, significantly correlated at 0.05 level. 


\subsection{Principal Component Analysis of Rice-eating Quality Traits}

To analyze the role of physiochemical indices in eating quality, AAC, GC, PC, ASV, and cooked rice taste value were selected for principal component analyses (Tables 7-9). For both low and medium AAC rice, the factor load matrix of PC and cooked rice taste value in factor 1 was remarkably higher than that of the other indices, indicating that PC was the most important factor for the eating quality of low and medium AAC rice. Factor 1 contributed to more than $30 \%$ of the factors for eating quality in both low and medium AAC rice. For total AAC rice, the factor load matrix of AAC, GC, and cooked rice taste value in factor 1 was remarkably higher than that of the other indices, indicating that AAC and GC were important factors for the eating quality of different type rice cultivars, contributing more than $43 \%$ to the rice-eating quality traits. The factor load matrix of PC in factor 2 was much higher than that of other indices, indicating the importance of $\mathrm{PC}$ in the eating quality of different type rice cultivars, contributing more than $22 \%$ to the rice eating quality traits.

Table 7. Factor load matrix of the eating quality of low AAC rice.

\begin{tabular}{lll}
\hline Variable & Factor $\mathbf{1}$ & Factor 2 \\
\hline AAC & -0.242 & 0.798 \\
GC & 0.578 & -0.286 \\
PC & -0.665 & -0.445 \\
ASV & 0.556 & -0.457 \\
Taste value & 0.638 & 0.496 \\
Eigenvalue & 1.551 & 1.371 \\
Percentage contribution $(\%)$ & 31.023 & 27.410 \\
Cumulative contribution $(\%)$ & 31.023 & 58.433 \\
\hline
\end{tabular}

Table 8. Factor load matrix of the eating quality of medium AAC rice.

\begin{tabular}{lll}
\hline Variable & Factor 1 & Factor 2 \\
\hline AAC & -0.425 & 0.227 \\
GC & 0.286 & 0.766 \\
PC & 0.815 & -0.053 \\
ASV & 0.112 & -0.783 \\
Taste value & -0.766 & -0.011 \\
Eigenvalue & 1.526 & 1.254 \\
Percentage contribution (\%) & 30.512 & 25.074 \\
Cumulative contribution (\%) & 30.512 & 55.586 \\
\hline
\end{tabular}

Table 9. Factor load matrix of the eating quality traits of total AAC rice.

\begin{tabular}{lll}
\hline Variable & Factor 1 & Factor 2 \\
\hline AAC & -0.875 & -0.148 \\
GC & 0.778 & 0.216 \\
PC & -0.134 & 0.926 \\
ASV & -0.336 & -0.311 \\
Taste value & 0.812 & -0.343 \\
Eigenvalue & 2.161 & 1.140 \\
Percentage contribution (\%) & 43.227 & 22.802 \\
Cumulative contribution (\%) & 43.227 & 66.029 \\
\hline
\end{tabular}

Association analysis of these indices and principal component analyses were performed to identify the important indices affecting the rice eating quality, which will assist in varietal selection in rice breeding. The principal component analysis was performed to identify the important factors of rice eating quality, and the results showed that $\mathrm{AAC}, \mathrm{GC}$, and cooked rice taste value were the most important factors affecting the eating quality of different type rice cultivars. Our results showed that for different japonica rice cultivars, the eating quality was largely determined by AAC and GC.

For low and medium AAC rice, the results of principal component analyses showed that PC and cooked rice taste value can be classified into one category; factor 1 , indicating that PC was the most important index for evaluating the eating quality of similar type rice cultivars. Furthermore, for total AAC rice, PC showed a significant negative correlation with cooked rice taste value, and its load matrix in factor 2 was high, indicating the importance of PC in different type rice cultivars. Thus, PC could be an essential indicator of eating quality in rice, especially for similar rice types.

Rice varieties with low GT save water and cooking time and tend to be selected by breeders [20]. The factor load matrix of ASV was not high in low and total AAC rice. Taken together, the results indicated GT may not be as important as AAC or $\mathrm{PC}$ for evaluating rice eating quality.

\section{Conclusion}

In total $\mathrm{AAC}$ rice, cooked rice taste value showed a significant negative correlation with AAC, CPV, SBV, and $\mathrm{CSV}$, and a positive correlation with GC, PKV, HPV, and BDV. PC showed a significant negative correlation with cooked rice taste value in low, medium, and total AAC rice. For both low and medium AAC rice, the factor load matrix of $\mathrm{PC}$ and cooked rice taste value in factor 1 was remarkably higher than that of the other indices, indicating that PC was the most important factor for the eating quality of low and medium AAC rice. For total AAC rice, the factor load matrix of $\mathrm{AAC}, \mathrm{GC}$, and cooked rice taste value in factor 1 was remarkably higher than that of the other indices, indicating that $\mathrm{AAC}$ and $\mathrm{GC}$ were important factors for the eating quality of different type rice cultivars. Taken together, AAC and $\mathrm{GC}$ were more important for the eating quality of different type rice cultivars; however, for similar type rice cultivars, PC was more important. It offers new insight into target traits in breeding rice with high eating quality. These findings and correlations of the present study will be useful for breeders during rice breeding selection for advanced generations.

\section{Acknowledgements}

We appreciate the financial supporting by the Shanghai Agriculture Applied Technology Development Program, China (Grant No. G2016060101), the outstanding Team Project from Shanghai Agricultural Academy of Science (Grant No. 2017A-05). 


\section{References}

[1] Nakamura, S., Suzuki, D., Kitadume, R., \& Ohtsubo, K. 2012. Quality Evaluation of Rice Crackers Based on Physicochemical Measurements. Biosci. Biotech. Biochem., 76, 794-804.

[2] Nakamura, S., Cui, J., Zhang, X., Yang, F., Xu, X., Sheng, H., \& Ohtsubo, K. 2016. Comparison of eating quality and physicochemical properties between Japanese and Chinese rice cultivars. Biosci. Biotech. Biochem., 80, 2437-2449.

[3] Chen, P., Shen, Z., Ming, L., Li, Y., Dan, W., Lou, G., Peng, B., Wu, B., Li, Y., Zhao, D., Gao, G., Zhang, Q., Xiao, J., Li, X., Wang, G., \& He, Y. 2018. Genetic basis of variation in rice seed storage protein (Albumin, Globulin, Prolamin, and Glutelin) content revealed by genome-wide association analysis. Front. Plant Sci., 9, 612.

[4] Xie, L., Chen, N., Duan, B., Zhu, Z., \& Liao, X. 2008. Impact of proteins on pasting and cooking properties of waxy and non-waxy rice. J. Cereal Sci., 47 (2), 372-379.

[5] Li, H., Yang, J., Yan, S., Lei, N., Wang, J., \& Sun, B. 2019. Molecular causes for the increased stickiness of cooked non-glutinous rice by enzymatic hydrolysis of the grain surface protein. Carbohydr. Polym., 216, 197-203.

[6] Wu, D. X., Shu, Q. Y., \& Xia, Y. W. 2011. Assisted Selection for early indica rice with good eating quality by RVA profile. Acta Agronomica Sinica, 27, 165-172.

[7] Hori, K., Suzuki, K., Iijima, K., \& Ebana, K. 2016. Variation in cooking and eating quality traits in Japanese rice germplasm accessions. Breeding Sci., 66, 309-318.

[8] Sun, M. M., Abdula, S. E., Lee, H. J., Cho, Y. C., Han, L. Z., Koh, H. J., \& Cho, Y. G. (2011. Molecular aspect of good eating quality formation in Japonica rice. Plos One, 6 (4), 112.

[9] Chen, M. H., Bergman, C. J., Pinson, S. R. M., Fjellstrom, R. G. 2008. Waxy gene haplotypes, Associations with pasting properties in an international rice germplasm collection. J. Cereal Sci., 48 (3), 81-88.

[10] Zhang, C., Chen, S., Ren, X., Lu, Y., Liu, D., Cai, X., Li, Q., Gao, J., \& Liu, Q. 2017. Molecular structure and physicochemical properties of starches from rice with different amylose contents resulting from modification of OsGBSSI activity. J. Agric. Food Chem., 65 (10), 2222-2232.

[11] Zhang, C., Zhu, L., Shao, K., Gu, M., \& Liu, Q. 2013. Toward underlying reasons for rice starches having low viscosity and high amylose: physiochemical and structural characteristics. J. Agric. Food Chem., 93, 1543-1551.

[12] Tan, YF., Li, J. X., Yu, S. B., Xing, Y. Z., Xu, C. G., \& Zhang, Q. F. 1999. The three important traits for cooking and eating quality of rice grains are controlled by a single locus in an elite rice hybrid, Shanyou 63. Theor Appl Genet., 99: 642-648.

[13] Tao, K., Li, C., Yu, W., Gilbert, R. G., \& Li, E. (2019. How amylose molecular fine structure of rice starch affects functional properties. Carbohydr. Polym., 204, 24-31.

[14] Sattari, A., Mahdinezhad, N., Fakheri, B., Noroozi, M., \& Beheshtizadeh, H. 2015. Improvement of the eating and cooking qualities of rice: a review. Int. J. Farming Allied Sci., 4, $153-160$.

[15] Tian, Z. X., Qian, Q., Liu, Q. Q., Yan, M. X., Liu, X. F., Yan, C. J., Liu, G. F., Gao, Z. Y., Tang, S. Z., Zeng, D. L., Wang, Y. H., Yu, J. M., Gu, M. H., \& Li, J. Y. 2009. Allelic diversities in rice starch biosynthesis lead to a diverse array of rice eating and cooking qualities. Proc. Nati. Acad. Sci., 106, 21760-21765.

[16] Ayabe, S., Kasai, M., Ohishi, K., \& Hatae, K. 2009. Textural properties and structures of starches from Indica and Japonica Rice with similar amylose content. Food Sci. Technol. Res., 15, 299-306.

[17] Sonoko, A., Midori, K., Kyoko, O., \& Keiko, H. 2009. Textural properties and structures of starches from Indica and Japonica rice with similar amylose content. Cereal Chem., 15, 299-306.

[18] Bao, J. S. 2012. Towards understanding of the genetic and molecular basis of eating and cooking quality of rice. Cereal Foods World, 57, 148-156.

[19] Wang, X., Pang, Y., Zhang, J., Wu, Z., Chen, K., Ali, J., Ye, G., $\mathrm{Xu}$, J., \& Li, Z. 2017. Genome-wide and gene-based association mapping for rice eating and cooking characteristics and protein content. Sci. Rep., 7, 17203.

[20] Waters, D. L., Henry, R. J., Reinke., R. F., \& Fitzgerald., M. A. 2006. Gelatinization temperature of rice explained by polymorphisms in starch synthase. Plant Biotechnol. J., 4 (1), 115-122. 